\title{
Meeting Communication Needs of Aged Patients with Cancer. A Continuous Challenge
}

Pavlos Sarafis $^{1^{*}}$ and Maria Malliarou ${ }^{2}$

${ }^{1}$ TEI Central Greece, Lamia, Greece

${ }^{2}$ Technological institution of thesallia, Greece

*Corresponding author: Pavlos Sarafis, TEl Central Greece, Lamia, Greece, Tel: +30 2410 684727; E-mail: psarafis@gmail.com

Received date: May 12, 2014, Accepted date: July 24, 2014, Published date: July 28, 2014

Copyright: (c) 2014 Sarafis $\mathrm{P}$, et al. This is an open-access article distributed under the terms of the Creative Commons Attribution License, which permits unrestricted use, distribution, and reproduction in any medium, provided the original author and source are credited.

\begin{abstract}
Introduction : The world population is aging and it is estimated that the number of older people with cancer will increase in the years ahead. Every patient communication needs are linked and related to their communication goals, in order to manage the cancer.
\end{abstract}

Aim : Of this literature review is to present the importance of meeting communication needs of older people with cancer.

Methodology : The present review is a literature review focusing on studies evaluating the communication needs of older people with cancer. Searches were conducted on MEDLINE for 10 years to 2014, using the following search terms: aged, older, people, patient, cancer, and need. Specific inclusion (reviews, research articles) discussing about communication needs of older people diagnosed with cancer have guided our review plan and the reviewed articles chosen were 16 manuscripts.

Results : Communication needs differs according to the age of the patients. Older people need their clinicians to recognize, appreciate and take into account the emotional and psychological impact of the diagnosis and treatment as well as being included in the decisions taken.

Conclusion : Good communication with older patients requires knowledge of their unmet needs. Nurses should be close to patients, discuss with them, stimulate them, especially older ones, and give them the opportunity to express their needs.

Keywords: Meeting communication; Oncology; Psychological impact

\section{Introduction}

The world population is aging and it is estimated that the number of older patients with cancer will increase in the years ahead [1]. Older people are entitled with special needs because they don't only have to face problems of the practice of oncology, but much more due to comorbidity, sensory, cognitive and physical deficits [2]. According to Hack et al. [3] every patient communication needs are linked and related to their communication goals, in order to manage the cancer (eg, knowing what the adverse effects of treatment are) and learn to live with it (eg, discussing emotional functioning or daily activities) Green et al furthermore argue that there is a greater possibility of the presence of cognitive impairment, loss of vision, and/or hearing loss in older patients compared with younger patients, which affects their ability to process and remember information [4,5]. In the United States, people over the age of 65 visit their doctor an average of eight times per year, compared to the general population's average of five visits per year [6]. Physicians should prepare for an increasing number of older patients by developing a greater understanding of this population and how to enhance communication with them. We need to remember that different patients have different communication needs, which may require different techniques [7].

The treatment options and adverse effects of cancer are complex. Patients with cancer are face crisis in their lives. Often, communication with those patients involves giving patients bad news. In order to meet cancer patient communication needs we have to be genuine with a caring presence. Healthcare personnel should show interest in the person, set the agenda to work collaboratively with the patient, aligning their expectations with those of the patient. Only then discuss the treatment options [8]. Training can help effectively address patient emotions. Every oncologist dreads communicating to patients that no additional treatment would be helpful.

Numerous studies make clear that improved communication improves patient satisfaction, promotes adherence to treatment, and reduces anxiety. Acquiring key communication skills will help in identifying patients' problems more accurately and give greater job satisfaction and less work-related stress [9]. Searching the literature techniques found to improve Physician-Patient Communication were "sitting at eye level with the patient and focusing on patient during the visit, after making an eye contact and communicating with short sentences. Simple language or drawings and pictures that explain, acknowledge the patient's feelings. 


\section{Methodology}

The present review focused on studies evaluating the needs of older patients with cancer. It is a synthesis and analysis of relevant published work that forms a literature review. Searches were conducted on MEDLINE for 10 years to 2014, using the following search terms: aged, patient, older, people, cancer, need. Searches were defined by both inclusion and exclusion criteria to capture as much of the relevant literature as possible while trying to exclude extraneous material. The steps included a determination of duplication in abstracts across databases and a review of article titles for relevance. The detailed exclusion criteria included the following: manual removal of duplicates, all papers of no relevance to communication needs.

The inclusion criteria were: Both qualitative and quantitative studies were included. Individual case reports and articles that reviewed the literature were excluded. The initial search of the literature in PubMed resulted in a total of 1,780 hits. Reviews of these articles determined that 1,520 studies were not relevant to the topic of this review but were more broadly related to cancer including biological aspects of cancer and clinical issues related to treatment as well as general communication issues. The remaining articles (reviews and research articles) seemed relevant to the focus on older people communication needs and being diagnosed with cancer. Some abstracts were further deleted because of their status as editorial letters or short commentaries, their lack of substantial contribution to the literature, or the vague nature of the abstract. A subset of articles was chosen for in-depth review.

Detailed examination of these showed that only 16 articles met all the inclusion criteria and specifically those that referred to the elderly. The other studies were excluded for one or more of the following reasons: (1) studies that did not focus on cancer patients' needs, (2) studies that did not discuss the older patients' needs, (3) studies evaluated professionals' needs and not the patients' needs, (4) studies focusing on the needs of individuals with no cancer history, (5) studies not focusing on aged patients, (6) age effect not discussed, and (7) article not available.

The limitation of this literature review is the complete reliance on previously published research and the availability of these studies using the method outlined in the search methodology and the appropriateness of these studies with the criteria of the selection/ exclusion procedure.

\section{Results}

The topic of communication with patient alone has been the subject of countless journal articles over many decades. The recent literature review has proven that specific communication styles are needed to meet elder patients' needs when being at the end of his life.

Mazor et al. [8] report that patients' needs include clinicians conveying hope and optimism about the prognosis and treatment outcomes, preparing patients with adequate explanations effective information to reduce patients' anxiety and distress; for months and sometimes years later. Reassurance and optimism helps to reduce fear and anxiety. Aged patients need their clinicians to recognize, appreciate and take into account the emotional and psychological impact of the diagnosis and treatment as well as being included in the decisions taken. Positive outcomes in cancer care, including older patient quality of life, satisfaction with care, and medical outcomes, can be influenced by effective and empathic communication with patients and families [10]. Additional literature suggests that effective communication can improve palliative care by alleviating anxieties, encouraging situational control and promoting quality of life for patients [11]. As such, communication has an important role in the provision of care to those facing palliative and end of life care suggesting some ways to deal with the difficulties that might occur during the communication or the revelation of ending. Some of the above mentioned ways to communicate with the elderly are: communicate honestly and truthfully, develop a compassionate bedside manner, treat others as you would want to be treated, provide empathic care, take the time needed to communicate, and determine patient information and decision-making preferences [12].

Clinical communication needs differs according to the age of the patients. Clinical communication needs are those required during interactions with patients as part of patient care. Aged patients, in particular those patients presenting with poor health status and low socio-economic support, may be at increased risk for not receiving adequate care and information and hence exclusion from the decision making [13]. Kawakami et al. [14] reported that the decision of not informing older patients is generally made by the family or medical staff.

It is known that in many different cultures cancer patients are not told the truth about their situation and exact diagnosis is revealed only to relatives. Though there many studies that concluded that the elderly patients wish to receive information and to know about their disease as adult patients [15]. Patient preferences for involvement in decision making differ and more specifically as literature review supports it declines with age, and vary with communication style and beliefs about participation. Some older patients could feel more comfortable with having to exhibit fewer conversational behaviors and when patients and doctors share similar beliefs about patient involvement [16]. Other studies proved that clinical communication to cancer patients plays a crucial role in the care offered, adaptation to the disease and the ability to cope with the disease [17] as information disclosure has been associated decreasing levels of anxiety, mood disorders and affective distress [18].

\section{Conclusion}

Older people with cancer reported less often that they needed information on treatment. More specifically, specific information and a week-by-week progress, what the treatment would exactly do and accomplish, seems to be relatively less important for older patients $[19,20]$.

As a conclusion older people have different needs for information on psychosocial issues [21]. Good communication with older patients requires knowledge of their unmet needs. Nurses should be close to patients, discuss with them, stimulate them, especially older ones, and give them the opportunity to express their needs. Greater numbers of cancer patients wish to be fully informed and involved in decision making, and effective communication is high on cancer patients' priorities for care. Attention to cultural needs that might mediate how much and to whom information is disclosed and discussed is important. Also promote an individualized plan of care with attainable and individualized goals for the elderly is needed [22].

\section{References}

1. Yancik R, Ries LA (2004) Cancer in older persons: an international issue in an aging world. Semin Oncol 31: 128-136. 
Citation: Sarafis P, Malliarou M (2014) Meeting Communication Needs of Aged Patients with Cancer. A Continuous Challenge. J Nurs Care 3: 185. doi:10.4172/2167-1168.1000185

Page 3 of 3

2. Repetto L, Fratino L, Audisio R.A, Venturino A, Gianni W et al. (2002) Comprehensive geriatric assessment adds information to Eastern Cooperative Oncology Group performance status in elderly cancer patients: An Italian Group for Geriatric Oncology study. Journal of Clinical Oncology 20: 494-502

3. Hack TF, Degner LF, Parker PA; SCRN Communication Team (2005) The communication goals and needs of cancer patients: a review. Psychooncology 14: 831-845.

4. Greene MG, Adelman RD (2003) Physician-older patient communication about cancer. Patient Educ Couns 50: 55-60.

5. Fitch MI, Gray RE, Franssen E (2001) Perspectives on living with ovarian cancer: older women's views. Oncol Nurs Forum 28: 1433-1442.

6. Thompson TL, Robinson JD, Beisecker AE (2004) The older patientphysician interaction. In: Nussbaum JF, Coupland J, eds. Handbook of Communication and Aging Research, 2nd ed. Mahwah, NJ: Lawrence Erlbaum Assoc.

7. Robinson TE 2nd, White GL Jr, Houchins JC (2006) Improving communication with older patients: tips from the literature. Fam Pract Manag 13: 73-78.

8. Mazor KM, Beard RL, Alexander GL, Arora NK, Firneno C et al. (2013) Patients' and family members' views on patient-centered communication during cancer care. Psychooncology. 22: 2487-2495

9. Chouliara Z, Kearney N, Stott D, Molassiotis A, Miller M (2004) Perceptions of older people with cancer of information, decision making and treatment: a systematic review of selected literature. Ann Oncol 15: 1596-1602.

10. Baile WF, Aaron J (2005) Patient-physician communication in oncology: past, present, and future. Curr Opin Oncol 17: 331-335.

11. Wallace P (2001) Improving palliative care through effective communication. Int J Palliat Nurs 7: 86-90.

12. Rodriguez KL, Young AJ (2005) Perspectives of elderly veterans regarding communication with medical providers about end-of-life care. J Palliat Med 8: 534-544.
13. Elkin EB, Kim SH, Casper ES, Kissane DW, Schrag D (2007) Desire for information and involvement in treatment decisions: elderly cancer patients' preferences and their physicians' perceptions. J Clin Oncol 25: 5275-5280.

14. Kawakami S, Arai G, Ueda K, Murai Y, Yokomichi H, et al. (2001) Physician's attitudes towards disclosure of cancer diagnosis to elderly patients: a report from Tokyo, Japan. Arch Gerontol Geriatr 33: 29-36.

15. Stewart M, Meredith L, Brown JB, Galajda J (2000) The influence of older patient-physician communication on health and health-related outcomes. Clin Geriatr Med 16: 25-36, vii-viii.

16. Schneider A, Körner T, Mehring M, Wensing M, Elwyn G, et al. (2006) Impact of age, health locus of control and psychological co-morbidity on patients' preferences for shared decision making in general practice. Patient Educ Couns 61: 292-298.

17. Butow PN, Maclean M, Dunn SM, Tattersall MH, Boyer MJ (1997) The dynamics of change: cancer patients' preferences for information, involvement and support. Ann Oncol 8: 857-863.

18. Annunziata MA, Foladore S, Magri MD, Crivellari D, Feltrin A, et al. (1998) Does the information level of cancer patients correlate with quality of life? A prospective study. Tumori 84: 619-623.

19. Pasetto LM, Falci C, Compostella A, Sinigaglia G, Rossi E, et al. (2007) Quality of life in elderly cancer patients. Eur J Cancer 43: 1508-1513.

20. Jesse Jansen, Julia van Weert, Sandra van Dulmen, Thea Heeren, Jozien Bensing (2007) Patient Education about Treatment in Cancer Care: An Overview of the Literature on Older Patients' Needs. Cancer Nurs. 30:251-260.

21. Wong F, Stewart DE, Dancey J, Meana M, McAndrews MP, et al. (2000) Men with prostate cancer: influence of psychological factors on informational needs and decision making. J Psychosom Res 49: 13-19.

22. Schulman-Green D, McCorkle R, Cherlin, E, Johnson-Hurzeler R, Bradley E.H (2005) Nurses' communication of prognosis and implications for hospice referral: a study of nurses caring for terminally ill hospitalized patients. American Journal of Critical Care 14: 64-70. 